\title{
喹啉酮和吡啶酮稠合的氧杂双环[3.3.1]壬烷的合成
}

\author{
叶明琰邱少中殷国栋* \\ (湖北师范大学化学化工学院 污染物分析与资源化湖北省重点实验室 黄石 435002)
}

\begin{abstract}
摘要 以 2-羊基查尔酮与 4-羟基- $2(1 H)$-喹啉酮或 4-羊基- $2(1 H)$-吡啶酮在正丙醇中回流反应，立体选择性地合成了喹啉 酮和吡啶酮稠合的 2,8-二氧杂双环 [3.3.1]壬烷衍生物. 其中, 喹啉酮片段通过烷基化反应可转化为相应的氮烷基取代的 喹啉酮和氧烷基取代的喹啉结构. 目标产物均未见文献报道, 其结构都通过了 ${ }^{1} \mathrm{H} N \mathrm{NMR} 、{ }^{13} \mathrm{C} N \mathrm{~N}$ 、 IR 和 HRMS 的表 征, 6-苯基-2,12-二氢-1 $H$-6, 12-亚甲基苯并 [7,8][1,3]二氧桥 [5,4-c]吡啶-1-酮(3r)和 1-异丙氧基-8-苯基-14H-8,14-亚甲基苯 并[7,8][1,3]二氧桥 [5,4-c]喹啉 (6c) 的结构和立体构型还进一步获得了 X 射线单晶衍射的证实.

关键词 4-差基-2(1H)-喹啉酮; 4-羊基-2(1H)-吡啶酮; 双环[3.3.1]壬烷; 烷基化; 单晶结构
\end{abstract}

\section{Synthesis of Quinolinone- and Pyridinone-Fused Oxabicyclo[3.3.1]nonanes}

\author{
Ye, Mingyan Qiu, Shaozhong Yin, Guodong* \\ (Hubei Key Laboratory of Pollutant Analysis and Reuse Technology, College of Chemistry and Chemical Engineering, \\ Hubei Normal University, Huangshi 435002)
}

\begin{abstract}
An efficient and stereoselective synthesis of quinolinone- and pyridinone-fused 2,8-dioxabicyclo[3.3.1]-nonanes by the reaction of 2-hydroxychalcones with 4-hydroxy-2(1H)-quinolinones/4-hydroxy-2(1H)-pyridinones in refluxing $n$-PrOH is described. The quinolinone fragment can be converted into the corresponding $N$-alkylated quinolinones and $O$-alkylated quinolines by the alkylation reaction. All the unknown compounds are characterized by means of ${ }^{1} \mathrm{H} N M R,{ }^{13} \mathrm{C} N M R$, IR and HRMS. The structures and configurations of 6-phenyl-2,12-dihydro- $1 H-6,12$-methanobenzo[7,8][1,3]dioxocino[5,4-c]pyridin-1-one (3r) and 1-isopropoxy-8-phenyl-14H-8,14-methanobenzo[7,8][1,3]dioxocino[5,4-c]quinoline (6c) are further confirmed by X-ray single crystal diffraction analysis.
\end{abstract}

Keywords 4-hydroxy-2(1H)-quinolinone; 4-hydroxy-2(1H)-pyridinone; bicyclo[3.3.1]nonane; alkylation; X-ray structure

2(1H)-喹啉酮和 2(1H)-吡啶酮是两类重要的杂环化 合物，其结构片段大量存在于许多天然的生物碱中，如 Euodenine A、Funiculosin、Ilicicolin $\mathrm{H}^{\text {等 }}{ }^{[1 \sim 4]}$. 含有这类 杂环骨架的分子常常展现出良好的抗菌、抗病毒和抗癌 活性 ${ }^{[5 \sim 7]}$. 4-差基-2(1H)-喹啉酮是一个含有多官能团的 分子, 羟基和氨基都容易参与反应, 3-位碳原子也具有 很好的亲核性, 因此被广泛应用于合成一些功能化的杂 环化合物 ${ }^{[8 \sim 10]}$. 此外, 双环[3.3.1]壬烷是一种具有 V-型 结构的迷人分子 ${ }^{[11,12]}$, 它们在药物化学和分子识别中表 现出潜在的应用价值 ${ }^{[13,14]}$, 因此, 这类化合物的制备与 功能化近年来受到了化学家的持续关注 ${ }^{[15 \sim 20]}$. 在最近 的研究中, 我们发展了一种以 2-羟基查尔酮与六元环状
的 1,3-二酩(或荎酚/取代苯酚)高立体选择性地合成 2,8二氧杂双环[3.3.1]壬烷的新方法 ${ }^{[21 ~ 23] . ~}$. 然而, 目前鲜有 文献引入含氮芳香环于此类结构中 ${ }^{[24]}$, 此处我们进一 步将反应底物拓展为 4-着基- $2(1 H)$-喹啉酮和 4-着基$2(1 H)$-吡啶酮衍生物, 立体选择性地合成了系列新型含 有喹啉酮和吡啶酮稠合的 2,8-二氧杂双环[3.3.1]壬烷, 并发现前者与卤代烷烃反应，喹啉酮片段可有效地转化 为相应的氮烷基取代的喹啉酮和氧烷基取代的喹啉结 构.

\section{1 结果与讨论}

在前面的工作中, 我们发现甲苯或正丙醇是合成香

\footnotetext{
* Corresponding authors. E-mail: gdyin@hbnu.edu.cn; gdyin_hbnu@163.com

Received September 26, 2016; revised November 3, 2016; published online November 29, 2016.

Project supported by the National Natural Science Foundation of China (No. 21542009).

国家自然科学基金(No. 21542009)资助项目.
} 
豆素稠合的 2,8-二氧杂双环[3.3.1]壬烷衍生物的理想溶 剂 $^{[21]}$. 考虑到反应底物的溶解性, 我们将 2-差基查尔酮 (1a, $0.5 \mathrm{mmol}$ )和 4-差基-2(1H)-喹啉酮 (2a, $0.5 \mathrm{mmol}$ )混 合于正丙醇中, 反应回流 $12 \mathrm{~h}$ 后反应原料消失, 冷却至 室温后，发现有大量的白色固体从溶液中析出，通过了 ${ }^{1} \mathrm{H} N M R 、{ }^{13} \mathrm{C}$ NMR、IR 和 HRMS 的表征, 证实其结构 为 8 -苯基- 2,14 -二氢- $1 H-8,14$-亚甲基苯并 $[7,8][1,3]$ 二氧 桥 $[5,4-c]$ 喹啉-1-酮(3a). 为了拓展该反应底物的范围, 我们尝试以各种取代的 2-羟基查尔酮 ${ }^{[25]}$ 与 4-羟基2(1H)-喹啉酮反应来考察该方法的通用性, 实验结果如 Eq. 1 所示. 当底物 1 中 $R^{1}$ 为氢原子, $R^{2}$ 为苯环上连接供 电子取代基(甲基、甲氧基), 相应的目标产物 $\mathbf{3 b} \sim \mathbf{3 d}$ 可 以较理想的收率分离得到. 另外, 当 $\mathrm{R}^{2}$ 为苯环上连接卤 原子(氟、氯和溴), 反应以 $68 \% \sim 76 \%$ 产率得到产物 $3 \mathrm{e} \sim$ 3g. 含有呋喃环、噻吩环和䒺环取代的底物也适合该反 应, $3 \mathbf{h} \sim 3 \mathbf{j}$ 的产率范围为 $65 \% \sim 83 \%$. 当 $\mathrm{R}^{2}$ 为甲基以及 2-甲基-1-丙烯基时, $3 \mathbf{k}$ 和 31 分别以 53\%和 51\%的产率分 离得到. 若底物中 $R^{2}$ 为苯环或取代苯环, $R^{1}$ 分别为 4溴、4-氯和 2,4-二氯取代的 2-羟基查尔酮时，产物 3m 30 也可以 $65 \% \sim 80 \%$ 的产率得到. 若 $R^{1}$ 和 $R^{3}$ 分别为氢 原子和甲基, $\mathrm{R}^{2}$ 为苯环以及 4-甲基取代苯环时，则以 $73 \%$ 和 75\% 的产率分离得到 $\mathbf{3 p}$ 和 $\mathbf{3 q}$.
随后，我们将底物 4-着基-2(1H)-喹啉酮拓展为一些 代表性的 2(1H)-吡啶酮，观察到 4-差基-2(1H)-吡啶酮 (2b)、4-羟基-6-甲基-2(1H)-吡啶酮(2c)和 4-差基-5-乙酯 基-2(1H)-吡啶酮(2d)也可以很好地与 2-羟基查尔酮(1a) 反应，以较好的产率生成相应的产物 $3 r \sim 3 t$, 其中 $3 r$ 的 结构得到了 $\mathrm{X}$ 射线单晶衍射的验证 (CCDC 号为: 1505511), 如图 1 所示. 4-差基-5-氯-2(1H)-吡啶酮(2e)俗 称吉莫斯特(Gimeracil), 是一种抗肿瘤药物, 主要用于 胃癌的治疗 ${ }^{[26]}$. 我们发现, 在标准反应条件下, $2 \mathrm{e}$ 与 $\mathbf{1 a}$ 也可以生成目标产物 $\mathbf{3 u}$.

氧烷基取代的喹啉也是一种重要的分子，含有这类 结构片段的化合物大量存在于天然产物和具有良好生 物活性分子中 ${ }^{[27]}$. 2(1H)-喹啉酮中存在酰胺键, $\mathrm{N}$ 原子上 的氢很容易被取代，我们也期望将上述得到的产物 $\mathbf{3 a}$ 进一步衍生化. 以碳酸钾为碱, 将溴代物 $\mathbf{4}$ 与 $\mathbf{3 a}$ 混合于 $N, N$-二甲基甲酰胺中反应，反应过程中发生了 $\mathrm{O}=\mathrm{CNH}$ 与 $\mathrm{C}=\mathrm{NOH}$ 异构化 ${ }^{[28]}$. 如 Eq. 2 所示, 当以溴乙烷和苄 溴为烷基化试剂时, 喹啉酮片段均转化为两种结构, 分 别为氮烷基取代的喹啉酮 $(5 \mathbf{a}, 5 \mathbf{b})$ 和氧烷基取代的喹啉 $(6 \mathbf{a}, 6 \mathbf{b})$, 且前者为主要产物 ${ }^{[29]}$. 然而, 当溴代异丙烷和 3a 反应时, 只是分离得到了氧烷基取代的喹啉稠合双
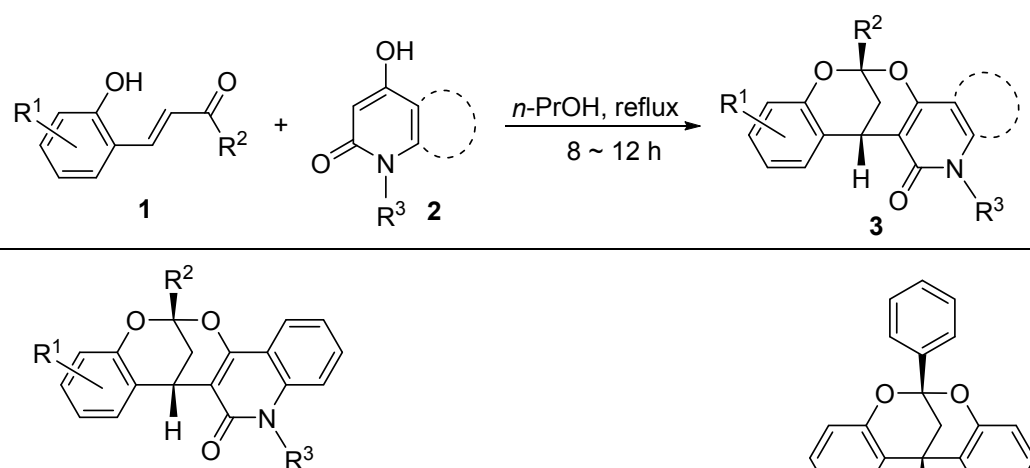

3a: $R^{1}=R^{3}=H, R^{2}=C_{6} H_{5}, 82 \%$ 3b: $R^{1}=R^{3}=H, R^{2}=4-M_{e} \mathrm{C}_{6} \mathrm{H}_{4}, 80 \%$ 3c: $\mathrm{R}^{1}=\mathrm{R}^{3}=\mathrm{H}, \mathrm{R}^{2}=4-\mathrm{MeOC}_{6} \mathrm{H}_{4}, 79 \%$ 3d: $\mathrm{R}^{1}=\mathrm{R}^{3}=\mathrm{H}, \mathrm{R}^{2}=3,4-(\mathrm{MeO})_{2} \mathrm{C}_{6} \mathrm{H}_{3}, 75 \%$ 3e: $R^{1}=R^{3}=H, R^{2}=4-F_{6} H_{4}, 68 \%$ 3f: $\mathrm{R}^{1}=\mathrm{R}^{3}=\mathrm{H}, \mathrm{R}^{2}=4-\mathrm{ClC}_{6} \mathrm{H}_{4}, 76 \%$ 3g: $R^{1}=R^{3}=H, R^{2}=4-B r C_{6} H_{4}, 72 \%$ 3h: $R^{1}=R^{3}=H, R^{2}=2$-Furyl, $65 \%$ 3i: $R^{1}=R^{3}=H, R^{2}=2$-Thienyl, $67 \%$ 3j: $R^{1}=R^{3}=H, R^{2}=1$-Naphthyl, $83 \%$ 3k: $R^{1}=R^{3}=H, R^{2}=M e, 53 \%$ 3I: $\mathrm{R}^{1}=\mathrm{R}^{3}=\mathrm{H}, \mathrm{R}^{2}=\mathrm{CH}=\mathrm{C}\left(\mathrm{CH}_{3}\right)_{2}, 51 \%$ $3 m: R^{1}=4-B r, R^{2}=C_{6} H_{5}, R^{3}=H, 79 \%$ 3n: $\mathrm{R}^{1}=4-\mathrm{Cl}, \mathrm{R}^{2}=4-\mathrm{MeOC}_{6} \mathrm{H}_{4}, \mathrm{R}^{3}=\mathrm{H}, 80 \%$ 3o: $R^{1}=2,4-d i C l, R^{2}=4-\mathrm{FC}_{6} \mathrm{H}_{4}, \mathrm{R}^{3}=\mathrm{H}, 65 \%$ 3p: $\mathrm{R}^{1}=\mathrm{H}, \mathrm{R}^{2}=\mathrm{C}_{6} \mathrm{H}_{5}, \mathrm{R}^{3}=\mathrm{Me}, 73 \%$ 3q: $R^{1}=H, R^{2}=4-M_{e} C_{6} H_{4}, R^{3}=M e, 75 \%$

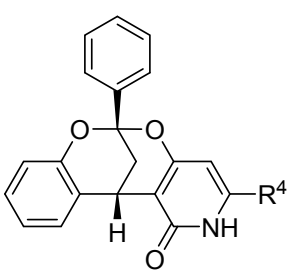

3r: $\mathrm{R}^{4}=\mathrm{H}, 78 \%$ 3s: $R^{4}=M e, 68 \%$

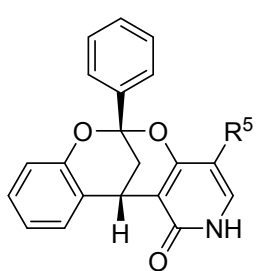

3t: $R^{5}=$ COOEt, $76 \%$ 3u: $\mathrm{R}^{5}=\mathrm{Cl}, 65 \%$

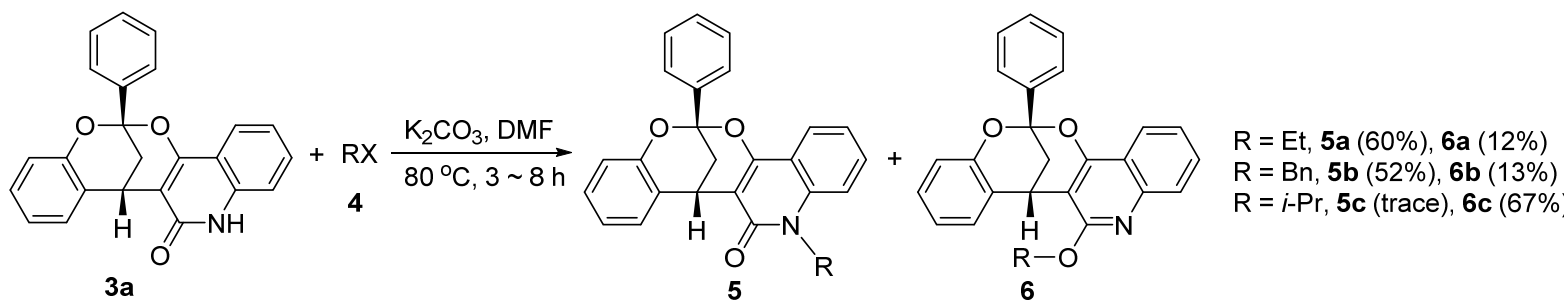




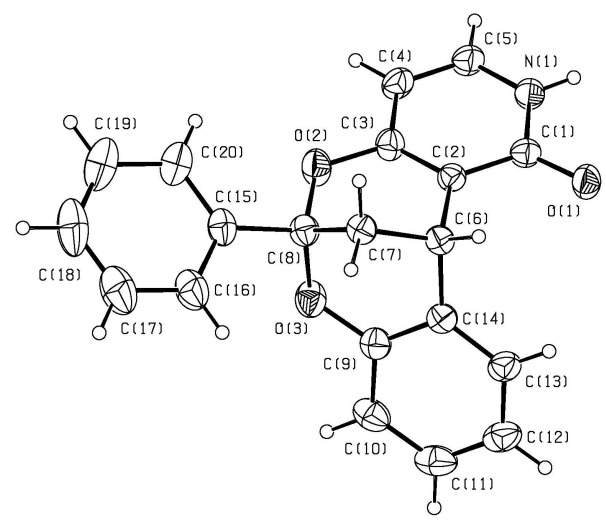

图 1 化合物 $\mathbf{3 r}$ 的晶体结构(省去了 $\mathrm{H}_{2} \mathrm{O}$ )

Figure 1 X-ray structure of compound $3 \mathbf{r}\left(\mathrm{H}_{2} \mathrm{O}\right.$ is omitted for clarity)

环 [3.3.1]壬烷产物(6c), 相应的氮烷基取代的产物 $5 \mathbf{c}$ 并 没有分离得到. 可能是由于异丙基立体因素影响, 在形 成氧烷基化时, 空间位阻更小的缘故. $6 \mathbf{c}$ 的结构也获得 了 $\mathrm{X}$ 射线单晶衍射的进一步证实(CCDC 号为 1505510), 如图 2 所示.

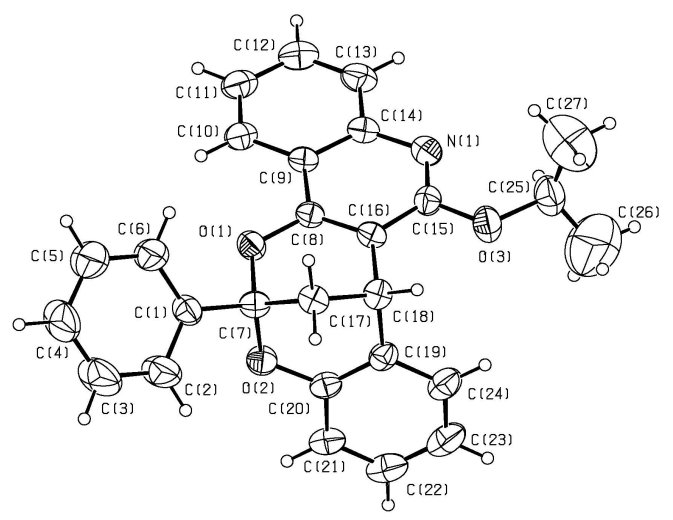

图 2 化合物 $6 \mathbf{c}$ 的晶体结构

Figure 2 X-ray structure of compound $\mathbf{6 c}$

\section{2 结论}

本文报道了一种简单有效合成喹啉酮和吡啶酮稠 合的 2,8-二氧杂双环[3.3.1]壬烷衍生物 3 的方法, 反应 将易得的 2-差基查尔酮(1)与 4-差基-2(1H)-喹啉酮或 4差基-2(1H)-吡啶酮(2)在正丙醇中回流既可以得到产物, 无需其它催化剂. 药物分子 4-着基-5-氯-2(1H)-吡啶酮 也成功地引入到目标产物中. 我们将 $\mathbf{3 a}$ 在碱性条件下 与卤代烷烃反应, 将喹啉酮片段转化成了相应的烷氮基 取代的喹啉酮和氧烷基取代的喹啉. 本文中合成的目标 产物均未见文献报道, 其结构都通过了 ${ }^{1} \mathrm{H} N \mathrm{NM} 、{ }^{13} \mathrm{C}$ NMR、IR 和 HRMS 的表征, 其中 $3 r$ 和 $6 \mathbf{c}$ 的结构和立 体构型还获得了 $\mathrm{X}$ 射线单晶衍射的证实.

\section{3 实验部分}

\section{1 仪器与试剂}

熔点采用泰克 X-4 型数字显示显微熔点仪测定，温 度未经校正; ${ }^{1} \mathrm{H}$ NMR 和 ${ }^{13} \mathrm{C}$ NMR 采用 Bruker AV 300 型核磁共振仪测定, $\mathrm{CDCl}_{3}$ 或 DMSO- $d_{6}$ 为溶剂, TMS 为 内标; IR 采用 Nicolet 5700 型红外分光光度仪测定 $(\mathrm{KBr}$ 压片); HRMS 采用 Bruker UltrafleXtreme MALDI $\mathrm{TOF} / \mathrm{TOF}$ [以 $\alpha$-氰基-4-羟基肉桂酸(HCCA)为基质]型高 分辨质谱仪测定; $X$ 射线单晶衍射是通过 Bruker SMART APEX CCD 测定.

所用试剂均为市售分析纯，未进一步纯化. 所用有 机溶剂在使用前均经过干燥并蒸馏提纯.

\section{2 实验方法}

\subsection{1 目标化合物 $\mathbf{3}$ 的合成}

将 2-羟基查尔酮类化合物 1 (0.5 mmol)和 4-差基2(1H)-吡啶酮衍生物 $2(0.5 \mathrm{mmol})$ 加入到 $25 \mathrm{~mL}$ 耐压管 中，再向其中加入正丙醇 $(5 \mathrm{~mL})$, 反应一段时间后有大 量固体析出. 当反应物消失后 $[8 \sim 12 \mathrm{~h}$, 用薄层色谱 (TLC)监测], 将混合物冷却至室温, 固体用少量无水乙 醇洗涤，抽滤，干燥，得到目标化合物 $\mathbf{3 a} \sim 3 \mathbf{3}$.

8-苯基-2,14-二氢- $1 H-8,14$-亚甲基苯并 $[7,8][1,3]$ 二 氧桥[5,4-c]喹啉-1-酮(3a): 白色固体 $151 \mathrm{mg}$, 产率 $82 \%$. m.p. $>300{ }^{\circ} \mathrm{C} ;{ }^{1} \mathrm{H}$ NMR (300 MHz, DMSO- $\left.d_{6}\right) \delta: 11.63$ $(\mathrm{s}, 1 \mathrm{H}), 7.83 \sim 7.80(\mathrm{~m}, 3 \mathrm{H}), 7.57 \sim 7.42(\mathrm{~m}, 5 \mathrm{H}), 7.30(\mathrm{~d}$, $J=8.1 \mathrm{~Hz}, 1 \mathrm{H}), 7.22 \sim 7.14(\mathrm{~m}, 2 \mathrm{H}), 7.02 \sim 6.92(\mathrm{~m}, 2 \mathrm{H})$, $4.39 \sim 4.37(\mathrm{~m}, 1 \mathrm{H}), 2.47(\mathrm{~d}, J=2.7 \mathrm{~Hz}, 2 \mathrm{H}) ;{ }^{13} \mathrm{C} \mathrm{NMR}$ $\left(75 \mathrm{MHz}, \mathrm{DMSO}-d_{6}\right) \delta$ : 161.0, 154.3, 151.4, 140.1, 137.3, $130.7,129.1,128.5,127.8,126.3,125.6,121.8,121.7$, $121.4,115.9,115.3,113.5,111.4,99.4,31.5,26.0$; IR (KBr) v: 3417, 2950, 1653, 1492, 1398, 1233, 1035, 989, $753,499 \mathrm{~cm}^{-1}$; HRMS (MALDI) calcd for $\mathrm{C}_{24} \mathrm{H}_{18} \mathrm{NO}_{3}$ $[\mathrm{M}+\mathrm{H}]^{+}$368.1281, found 368.1280.

8-(4- 甲基苯基)-2,14-二氢- $1 H$-8, 14- 亚甲基苯并 [7,8][1,3]二氧桥[5,4-c]喹啉-1-酮(3b): 白色固体 $153 \mathrm{mg}$, 产率 80\%. m.p. $>300{ }^{\circ} \mathrm{C} ;{ }^{1} \mathrm{H}$ NMR $\left(300 \mathrm{MHz}, \mathrm{DMSO}-d_{6}\right)$ $\delta$ : $11.62(\mathrm{~s}, 1 \mathrm{H}), 7.80(\mathrm{~d}, J=7.9 \mathrm{~Hz}, 1 \mathrm{H}), 7.69$ (d, $J=7.7$ $\mathrm{Hz}, 2 \mathrm{H}), 7.53 \sim 7.42(\mathrm{~m}, 2 \mathrm{H}), 7.35 \sim 7.29(\mathrm{~m}, 3 \mathrm{H}), 7.18(\mathrm{q}$, $J=7.9 \mathrm{~Hz}, 2 \mathrm{H}), 7.01 \sim 6.92(\mathrm{~m}, 2 \mathrm{H}), 4.37(\mathrm{~s}, 1 \mathrm{H}), 2.50(\mathrm{~s}$, $2 \mathrm{H}), 2.38(\mathrm{~s}, 3 \mathrm{H}) ;{ }^{13} \mathrm{C}$ NMR $\left(75 \mathrm{MHz}, \mathrm{DMSO}-d_{6}\right) \delta: 161.0$, $154.3,151.4,138.5,137.32$, 137.27, 130.6, 129.0, 127.8, $126.3,125.5,121.71,121.68,121.3,115.8,115.3,113.5$, 111.3, 99.4, 31.5, 26.1, 20.8; IR (KBr) v: 3434, 2945, 1653, 1491, 1398, 1229, 1034, 992, 861, $750 \mathrm{~cm}^{-1}$; HRMS (MALDI) calcd for $\mathrm{C}_{25} \mathrm{H}_{20} \mathrm{NO}_{3}[\mathrm{M}+\mathrm{H}]^{+} 382.1438$, found 
382.1440 .

8-(4-甲氧基苯基)-2,14-二氢- $1 H$-8, 14-亚甲基苯并 [7,8][1,3]二氧桥[5,4-c]喹啉-1-酮(3c): 白色固体 $157 \mathrm{mg}$, 产率 79\%. m.p. $>300{ }^{\circ} \mathrm{C} ;{ }^{1} \mathrm{H}$ NMR $\left(300 \mathrm{MHz}, \mathrm{CDCl}_{3}\right) \delta$ : 11.17 (s, 1H), 7.98 (d, $J=7.4 \mathrm{~Hz}, 1 \mathrm{H}), 7.73$ (d, $J=8.9 \mathrm{~Hz}$, 2H), $7.63(\mathrm{dd}, J=7.5,1.3 \mathrm{~Hz}, 1 \mathrm{H}), 7.54 \sim 7.49(\mathrm{~m}, 1 \mathrm{H})$, $7.35(\mathrm{~d}, J=8.1 \mathrm{~Hz}, 1 \mathrm{H}), 7.23 \sim 7.12(\mathrm{~m}, 2 \mathrm{H}), 7.02(\mathrm{~d}, J=$ $8.8 \mathrm{~Hz}, 3 \mathrm{H}), 6.94(\mathrm{t}, J=7.4 \mathrm{~Hz}, 1 \mathrm{H}), 4.61 \sim 4.59(\mathrm{~m}, 1 \mathrm{H})$, $3.88(\mathrm{~s}, 3 \mathrm{H}), 2.50 \sim 2.35(\mathrm{~m}, 2 \mathrm{H}) ;{ }^{13} \mathrm{C} \mathrm{NMR}(75 \mathrm{MHz}$, $\left.\mathrm{CDCl}_{3}\right) \delta: 163.1,160.2,156.3,151.8,137.1,132.8,130.6$, $128.1,127.9,127.1,126.0,122.6,122.2,121.6,116.2$, $115.7,114.8,113.8,111.1,99.7,55.4,33.1,26.8$; IR (KBr) $v: 3436,2946,1652,1510,1443,1244,1102,1033,999$, $750 \mathrm{~cm}^{-1}$; HRMS (MALDI) calcd for $\mathrm{C}_{25} \mathrm{H}_{20} \mathrm{NO}_{4}[\mathrm{M}+$ $\mathrm{H}]^{+}$398.1387, found 398.1394.

8-(3,4-二甲氧基苯基)-2,14-二氢- $1 H-8,14$-亚甲基苯 并[7,8][1,3]二氧桥[5,4-c]喹啉-1-酮(3d): 白色固体 160 $\mathrm{mg}$, 产率 75\%. m.p. $>300{ }^{\circ} \mathrm{C} ;{ }^{1} \mathrm{H}$ NMR $(300 \mathrm{MHz}$, $\left.\mathrm{CDCl}_{3}\right) \delta: 11.16(\mathrm{~s}, 1 \mathrm{H}), 7.98(\mathrm{~d}, J=8.1 \mathrm{~Hz}, 1 \mathrm{H}), 7.64(\mathrm{~d}$, $J=6.3 \mathrm{~Hz}, 1 \mathrm{H}), 7.53(\mathrm{t}, J=7.1 \mathrm{~Hz}, 1 \mathrm{H}), 7.39 \sim 7.34(\mathrm{~m}$, $2 \mathrm{H}), 7.31(\mathrm{~d}, J=2.0 \mathrm{~Hz}, 1 \mathrm{H}), 7.25 \sim 7.13(\mathrm{~m}, 2 \mathrm{H}), 7.05 \sim$ $6.93(\mathrm{~m}, 3 \mathrm{H}), 4.61(\mathrm{~s}, 1 \mathrm{H}), 3.96(\mathrm{~s}, 6 \mathrm{H}), 2.52 \sim 2.37(\mathrm{~m}$, $2 \mathrm{H}) ;{ }^{13} \mathrm{C}$ NMR $\left(75 \mathrm{MHz}, \mathrm{CDCl}_{3}\right) \delta: 163.2,156.3,151.8$, $149.7,148.9,137.1,133.1,130.6,128.2,127.9,126.1$, $122.5,122.3,121.7,118.3,116.2,115.8,114.8,111.1$, $110.8,109.2,99.7,56.1,56.0,33.0,26.8$; IR (KBr) $v$ : 3430, 3001, 1653, 1513, 1345, 1133, 1098, 1025, 752 $\mathrm{cm}^{-1}$; HRMS (MALDI) calcd for $\mathrm{C}_{26} \mathrm{H}_{22} \mathrm{NO}_{5}[\mathrm{M}+\mathrm{H}]^{+}$ 428.1492, found 428.1492 .

8-(4-氟苯基)-2,14-二氢 - $1 H-8,14$ - 亚甲基苯并 [7,8][1,3]二氧桥[5,4-c]喹啉-1-酮(3e): 白色固体 $131 \mathrm{mg}$, 产率 68\%. m.p.>300 ${ }^{\circ} \mathrm{C} ;{ }^{1} \mathrm{H}$ NMR $\left(300 \mathrm{MHz}, \mathrm{DMSO}-d_{6}\right)$ $\delta: 11.63(\mathrm{~s}, 1 \mathrm{H}), 7.90 \sim 7.79(\mathrm{~m}, 3 \mathrm{H}), 7.54 \sim 7.48(\mathrm{~m}, 1 \mathrm{H})$, $7.45(\mathrm{~d}, J=1.4 \mathrm{~Hz}, 1 \mathrm{H}), 7.42 \sim 7.29(\mathrm{~m}, 3 \mathrm{H}), 7.18$ (q, $J=$ $7.4 \mathrm{~Hz}, 2 \mathrm{H}), 7.02 \sim 6.92(\mathrm{~m}, 2 \mathrm{H}), 4.39 \sim 4.37(\mathrm{~m}, 1 \mathrm{H}) 2.51$ $(\mathrm{s}, 2 \mathrm{H}) ;{ }^{13} \mathrm{C}$ NMR $\left(75 \mathrm{MHz}, \mathrm{DMSO}-d_{6}\right) \delta: 162.4\left(\mathrm{~d},{ }^{1} J_{\mathrm{C}-\mathrm{F}}=\right.$ $244.0 \mathrm{~Hz}), 160.9,154.2,151.3,137.3,136.5\left(\mathrm{~d},{ }^{4} J_{\mathrm{C}-\mathrm{F}}=3.0\right.$ $\mathrm{Hz}), 130.7,128.1\left(\mathrm{~d},{ }^{3} J_{\mathrm{C}-\mathrm{F}}=8.9 \mathrm{~Hz}\right), 127.8,126.2,121.74$, $121.69,121.4,115.8,115.30\left(\mathrm{~d},{ }^{2} J_{\mathrm{C}-\mathrm{F}}=21.5 \mathrm{~Hz}\right), 115.26$, 113.4, 111.4, 99.1, 31.3, 26.0; IR (KBr) v: 3432, 2850, 1653, 1502, 1399, 1231, 1101, 1003, $835 \mathrm{~cm}^{-1}$; HRMS (MALDI) calcd for $\mathrm{C}_{24} \mathrm{H}_{17} \mathrm{FNO}_{3}[\mathrm{M}+\mathrm{H}]^{+}$386.1187, found 386.1186 .

8-(4- 氯苯基)-2,14-二氢- $1 H-8,14$ - 亚甲基苯并 [7,8][1,3]二氧桥[5,4-c]喹啉-1-酮(3f): 白色固体 $153 \mathrm{mg}$,
产率 76\%. m.p. $>300 \quad{ }^{\circ} \mathrm{C} ;{ }^{1} \mathrm{H}$ NMR $\left(300 \mathrm{MHz}, \mathrm{DMSO}-d_{6}\right)$ $\delta: 11.65(\mathrm{~s}, 1 \mathrm{H}), 7.86 \sim 7.79(\mathrm{~m}, 3 \mathrm{H}), 7.61(\mathrm{~d}, J=8.4 \mathrm{~Hz}$, 2H), 7.51 (t, $J=7.6 \mathrm{~Hz}, 1 \mathrm{H}), 7.43$ (d, $J=7.4 \mathrm{~Hz}, 1 \mathrm{H}), 7.30$ $(\mathrm{d}, J=8.2 \mathrm{~Hz}, 1 \mathrm{H}), 7.18(\mathrm{q}, J=8.1 \mathrm{~Hz}, 2 \mathrm{H}), 7.03 \sim 6.93$ $(\mathrm{m}, 2 \mathrm{H}), 4.38(\mathrm{~s}, 1 \mathrm{H}), 2.48(\mathrm{~s}, 2 \mathrm{H}) ;{ }^{13} \mathrm{C} \mathrm{NMR}(75 \mathrm{MHz}$, DMSO- $\left.d_{6}\right) \delta: 160.9,154.2,151.2,139.1,137.3,133.9$, $130.7,128.6,127.9,127.8,126.2,121.8,121.7,121.5$, $115.9,115.3,113.4,111.4,99.1,31.2,25.9$; IR (KBr) $v$ : 3727, 3424, 2860, 1663, 1435, 1328, 1094, 881, $863 \mathrm{~cm}^{-1}$; HRMS (MALDI) calcd for $\mathrm{C}_{24} \mathrm{H}_{17} \mathrm{ClNO}_{3}[\mathrm{M}+\mathrm{H}]^{+}$ 402.0891, found 402.0890.

8-(4-溴苯基)-2,14-二氢- $1 H$-8, 14-亚甲基苯并 [7,8][1,3]二氧桥[5,4-c]喹啉-1-酮(3g): 白色固体 $161 \mathrm{mg}$, 产 率 72\%. m.p. $>300{ }^{\circ} \mathrm{C} ;{ }^{1} \mathrm{H} \mathrm{NMR}\left(300 \mathrm{MHz}, \mathrm{DMSO}-d_{6}\right) \delta$ : $11.63(\mathrm{~s}, 1 \mathrm{H}), 7.82 \sim 7.73(\mathrm{~m}, 5 \mathrm{H}), 7.53 \sim 7.43(\mathrm{~m}, 2 \mathrm{H})$, $7.32 \sim 7.29(\mathrm{~m}, 1 \mathrm{H}), 7.22 \sim 7.17(\mathrm{~m}, 2 \mathrm{H}), 7.02 \sim 6.95(\mathrm{~m}$, 2H), $4.38(\mathrm{~s}, 1 \mathrm{H}), 2.50 \sim 2.48(\mathrm{~m}, 2 \mathrm{H}) ;{ }^{13} \mathrm{C} \mathrm{NMR}(75 \mathrm{MHz}$, DMSO- $\left.d_{6}\right) \delta: 160.9,154.1,151.2,139.5,137.3,131.4$, $130.6,128.0,127.8,126.2,122.5,121.71,121.66,121.4$, $115.8,115.2,113.4,111.3,99.1,31.1,25.9$; IR (KBr) $v$ : 3442, 2850, 1651, 1489, 1394, 1230, 1101, 1034, 749 $\mathrm{cm}^{-1}$; HRMS (MALDI) calcd for $\mathrm{C}_{24} \mathrm{H}_{17} \mathrm{BrNO}[\mathrm{M}+\mathrm{H}]^{+}$ 446.0386, found 446.0392 .

8-(2-呋喃基)-2,14-二氢 - $1 H-8,14$ - 亚甲基苯并 [7,8][1,3]二氧桥[5,4-c]喹啉-1-酮(3h): 白色固体 $115 \mathrm{mg}$, 产率 65\%. m.p. $>300{ }^{\circ} \mathrm{C} ;{ }^{1} \mathrm{H}$ NMR (300 MHz, DMSO- $\left.d_{6}\right)$ $\delta: 11.65(\mathrm{~s}, 1 \mathrm{H}), 7.85 \sim 7.79(\mathrm{~m}, 2 \mathrm{H}), 7.53 \sim 7.44(\mathrm{~m}, 1 \mathrm{H})$, $7.41(\mathrm{~d}, J=1.2 \mathrm{~Hz}, 1 \mathrm{H}), 7.29(\mathrm{~d}, J=8.1 \mathrm{~Hz}, 1 \mathrm{H}), 7.22 \sim$ $7.12(\mathrm{~m}, 2 \mathrm{H}), 7.00 \sim 6.92(\mathrm{~m}, 3 \mathrm{H}), 6.64 \sim 6.63(\mathrm{~m}, 1 \mathrm{H})$, $4.41 \sim 4.40(\mathrm{~m}, 1 \mathrm{H}), 2.62 \sim 2.57(\mathrm{~m}, 2 \mathrm{H}) ;{ }^{13} \mathrm{C} \mathrm{NMR}(75$ $\left.\mathrm{MHz}, \mathrm{DMSO}-d_{6}\right) \delta: 160.8,153.6,151.0,150.8,143.8$, 137.2 , 130.7, 127.9, 126.1, 121.8, 121.5, 115.8, 115.3, $113.2,111.4,110.7,108.2,95.7,28.7,25.2$; IR (KBr) v: 3428, 2850, 2357, 1654, 1397, 1103, 961, 884, $750 \mathrm{~cm}^{-1}$; HRMS (MALDI) calcd for $\mathrm{C}_{22} \mathrm{H}_{16} \mathrm{NO}_{4}[\mathrm{M}+\mathrm{H}]{ }^{+}$ 358.1074, found 358.1072.

8-(2-噻吩基)-2,14-二氢 - $1 H-8,14$ - 亚甲基苯并 [7,8][1,3]二氧桥[5,4-c]喹啉-1-酮(3i): 白色固体 $125 \mathrm{mg}$, 产率 67\%. m.p. $>300{ }^{\circ} \mathrm{C} ;{ }^{1} \mathrm{H}$ NMR $\left(300 \mathrm{MHz}, \mathrm{DMSO}-d_{6}\right)$ $\delta: 11.65(\mathrm{~d}, J=1.9 \mathrm{~Hz}, 1 \mathrm{H}), 7.77$ (d, $J=8.0 \mathrm{~Hz}, 1 \mathrm{H}), 7.71$ $(\mathrm{d}, J=3.8 \mathrm{~Hz}, 1 \mathrm{H}), 7.70 \sim 7.48(\mathrm{~m}, 2 \mathrm{H}), 7.43(\mathrm{~d}, J=7.8$ $\mathrm{Hz}, 1 \mathrm{H}), 7.31 \sim 7.28(\mathrm{~m}, 1 \mathrm{H}), 7.23 \sim 7.13(\mathrm{~m}, 3 \mathrm{H}), 6.97 \sim$ $6.93(\mathrm{~m}, 2 \mathrm{H}), 4.40(\mathrm{~s}, 1 \mathrm{H}), 2.71 \sim 2.61(\mathrm{~m}, 2 \mathrm{H}) ;{ }^{13} \mathrm{C} \mathrm{NMR}$ $\left(75 \mathrm{MHz}, \mathrm{DMSO}-d_{6}\right) \delta$ : 160.9, 153.9, 151.0, 143.1, 137.3, $130.8,127.9,127.3,127.0,126.1,125.8,121.9,121.6$, 
$115.9,115.4,113.3,111.5,98.4,31.5,26.0$; IR (KBr) v: 3434, 2852, 1651, 1492, 1397, 1101, 986, 877, $755 \mathrm{~cm}^{-1}$; HRMS (MALDI) calcd for $\mathrm{C}_{22} \mathrm{H}_{16} \mathrm{NO}_{3} \mathrm{~S}[\mathrm{M}+\mathrm{H}]^{+}$ 374.0845 , found 374.0842 .

8-(1-菜基)-2,14-二氢- $1 H-8,14$-亚甲基苯并 [7,8][1,3] 二氧桥 $[5,4-c]$ 喹啉-1-酮 $(3 \mathbf{j})$ : 白色固体 $173 \mathrm{mg}$, 产率 $83 \%$. m.p. $>300{ }^{\circ} \mathrm{C} ;{ }^{1} \mathrm{H}$ NMR (300 MHz, $\left.\mathrm{CDCl}_{3}\right) \delta: 10.86$ $(\mathrm{s}, 1 \mathrm{H}), 8.41(\mathrm{~d}, J=8.3 \mathrm{~Hz}, 1 \mathrm{H}), 8.11(\mathrm{~d}, J=6.5 \mathrm{~Hz}, 1 \mathrm{H})$, $7.99 \sim 7.95(\mathrm{~m}, 2 \mathrm{H}), 7.91(\mathrm{~d}, J=7.4 \mathrm{~Hz}, 1 \mathrm{H}), 7.70(\mathrm{~d}, J=$ $1.4 \mathrm{~Hz}, 1 \mathrm{H}), 7.68 \sim 7.58(\mathrm{~m}, 2 \mathrm{H}), 7.55 \sim 7.47(\mathrm{~m}, 2 \mathrm{H}), 7.34$ $(\mathrm{d}, J=8.1 \mathrm{~Hz}, 1 \mathrm{H}), 7.21 \sim 7.15(\mathrm{~m}, 2 \mathrm{H}), 7.04 \sim 6.97(\mathrm{~m}$, $2 \mathrm{H}), 4.71 \sim 4.69(\mathrm{~m}, 1 \mathrm{H}), 2.87 \sim 2.72(\mathrm{~m}, 2 \mathrm{H}) ;{ }^{13} \mathrm{C} \mathrm{NMR}$ $\left(75 \mathrm{MHz}, \mathrm{CDCl}_{3}\right) \delta: 163.3,155.9,151.4,137.2,135.3$, $134.8,130.7,130.5,129.1,128.2,128.1,126.4,125.74$, $125.66,124.8,124.3,122.7,122.3,121.8,116.4,115.8$, $114.8,111.4,100.8,31.0,26.4$; IR (KBr) v: 3407, 2853, 1650, 1495, 1398, 1290, 1113, 938, $750 \mathrm{~cm}^{-1}$; HRMS (MALDI) calcd for $\mathrm{C}_{28} \mathrm{H}_{20} \mathrm{NO}_{3}[\mathrm{M}+\mathrm{H}]^{+} 418.1438$, found 418.1437 .

8-甲基-2,14-二氢- $1 H$-8,14-亚甲基苯并 $[7,8][1,3]$ 二 氧桥[5,4-c]喹啉-1-酮(3k): 白色固体 $81 \mathrm{mg}$, 产率 53\%. m.p. $>300{ }^{\circ} \mathrm{C} ;{ }^{1} \mathrm{H}$ NMR $\left(300 \mathrm{MHz}, \mathrm{CDCl}_{3}\right) \delta: 10.16$ (s, $1 \mathrm{H}), 7.91(\mathrm{~d}, J=8.2 \mathrm{~Hz}, 1 \mathrm{H}), 7.54(\mathrm{~d}, J=7.6 \mathrm{~Hz}, 1 \mathrm{H})$, $7.50 \sim 7.44(\mathrm{~m}, 1 \mathrm{H}), 7.22 \sim 7.16(\mathrm{~m}, 2 \mathrm{H}), 7.11 \sim 7.05(\mathrm{~m}$, $1 \mathrm{H}), 6.91 \sim 6.85(\mathrm{~m}, 2 \mathrm{H}), 4.50 \sim 4.48(\mathrm{~m}, 1 \mathrm{H}), 2.34 \sim 2.18$ $(\mathrm{m}, 2 \mathrm{H}), 2.00(\mathrm{~s}, 3 \mathrm{H}) ;{ }^{13} \mathrm{C} \mathrm{NMR}\left(75 \mathrm{MHz}, \mathrm{CDCl}_{3}\right) \delta$ : $162.6,156.1,151.5,136.7,130.5,128.1,127.7,125.9$, $122.7,122.1,121.3,115.9,115.2,114.7,111.0,99.0,30.8$, 26.9, 26.2; IR (KBr) v: 3432, 3065, 2942, 1653, 1489, 1447, 1149, 1086, $809 \mathrm{~cm}^{-1}$; HRMS (MALDI) calcd for $\mathrm{C}_{19} \mathrm{H}_{16} \mathrm{NO}_{3}:[\mathrm{M}+\mathrm{H}]^{+}$306.1125, found 306.1123.

8-(2-甲基-1-丙烯基)-2,14-二氢- $1 H$-8,14-亚甲基苯 并 $[7,8][1,3]$ 二氧桥 $[5,4-c]$ 喹啉-1-酮(31): 白色固体 88 $\mathrm{mg}$, 产率 51\%. m.p. $>300{ }^{\circ} \mathrm{C} ;{ }^{1} \mathrm{H}$ NMR $(300 \mathrm{MHz}$, $\left.\mathrm{CDCl}_{3}\right) \delta: 10.68(\mathrm{~s}, 1 \mathrm{H}), 7.93(\mathrm{~d}, J=0.9 \mathrm{~Hz}, 1 \mathrm{H}), 7.91$ (d, $J=1.0 \mathrm{~Hz}, 1 \mathrm{H}), 7.57 \sim 7.50(\mathrm{~m}, 1 \mathrm{H}), 7.28(\mathrm{~s}, 1 \mathrm{H}), 7.19(\mathrm{t}$, $J=8.0 \mathrm{~Hz}, 1 \mathrm{H}), 7.12 \sim 7.07(\mathrm{~m}, 1 \mathrm{H}), 6.90(\mathrm{t}, J=7.7 \mathrm{~Hz}$, $2 \mathrm{H}), 5.80(\mathrm{~s}, 1 \mathrm{H}), 4.50 \sim 4.48(\mathrm{~m}, 1 \mathrm{H}), 2.39 \sim 2.23(\mathrm{~m}$, 2H), $1.92(\mathrm{~s}, 6 \mathrm{H}) ;{ }^{13} \mathrm{C} \mathrm{NMR}\left(75 \mathrm{MHz}, \mathrm{CDCl}_{3}\right) \delta: 163.3$, $156.1,151.5,140.7,137.1,130.4,128.1,127.8,126.2$, $124.2,122.6,122.1,121.4,116.1,115.7,114.8,111.0$, 99.1, 30.5, 26.9, 26.2, 19.3; IR (KBr) v: 3436, 2852, 1649, 1441, 1399, 1100, 957, 880, $752 \mathrm{~cm}^{-1}$; HRMS (MALDI) calcd for $\mathrm{C}_{22} \mathrm{H}_{20} \mathrm{NO}_{3}[\mathrm{M}+\mathrm{H}]^{+}$346.1438, found 346.1432.

12-溴-8-苯基-2,14-二氢-1H-8,14-亚甲基苯并
$[7,8][1,3]$ 二氧桥[5,4-c]喹啉-1-酮(3m): 白色固体 176 $\mathrm{mg}$, 产率 79\%. m.p. $>300{ }^{\circ} \mathrm{C} ;{ }^{1} \mathrm{H}$ NMR $(300 \mathrm{MHz}$, DMSO- $\left.d_{6}\right) \delta: 11.69(\mathrm{~s}, 1 \mathrm{H}), 7.83 \sim 7.81(\mathrm{~m}, 3 \mathrm{H}), 7.58 \sim$ $7.49(\mathrm{~m}, 5 \mathrm{H}), 7.36 \sim 7.31(\mathrm{~m}, 2 \mathrm{H}), 7.22(\mathrm{t}, J=7.6 \mathrm{~Hz}, 1 \mathrm{H})$, $7.01(\mathrm{~d}, J=8.7 \mathrm{~Hz}, 1 \mathrm{H}), 4.38(\mathrm{~s}, 1 \mathrm{H}), 2.51(\mathrm{~s}, 2 \mathrm{H}) ;{ }^{13} \mathrm{C}$ NMR (75 MHz, DMSO- $\left.d_{6}\right) \delta: 160.9,154.4,150.9,139.7$, $137.4,130.9,130.4,129.9,129.3,128.9,128.6,125.7$, $121.9,121.8,118.2,115.4,113.4,112.6,110.7,99.6,30.9$, 25.9; IR (KBr) v: 3434, 2818, 1652, 1488, 1402, 1243, $1102,949,753 \mathrm{~cm}^{-1}$; HRMS (MALDI) calcd for $\mathrm{C}_{24} \mathrm{H}_{17^{-}}$ $\mathrm{BrNO}_{3}[\mathrm{M}+\mathrm{H}]^{+}$446.0386, found 446.0391.

12-氯-8-(4-甲氧基苯基)-2,14-二氢- $1 H-8,14$-亚甲基 苯并 $[7,8][1,3]$ 二氧桥 $[5,4-c]$ 喹啉-1-酮 $(3 n)$ : 白色固体 $172 \mathrm{mg}$, 产率 80\%. m.p.>300 ${ }^{\circ} \mathrm{C}$; ${ }^{1} \mathrm{H}$ NMR $(300 \mathrm{MHz}$, DMSO- $\left.d_{6}\right) \delta: 11.65(\mathrm{~s}, 1 \mathrm{H}), 7.81 \sim 7.72(\mathrm{~m}, 3 \mathrm{H}), 7.52 \sim$ $7.49(\mathrm{~m}, 1 \mathrm{H}), 7.41(\mathrm{~s}, 1 \mathrm{H}), 7.32$ (d, $J=8.2 \mathrm{~Hz}, 1 \mathrm{H}), 7.20$ $(\mathrm{s}, 2 \mathrm{H}), 7.08 \sim 7.02(\mathrm{~m}, 3 \mathrm{H}), 4.36(\mathrm{~s}, 1 \mathrm{H}), 3.82(\mathrm{~s}, 3 \mathrm{H})$, 2.47 (s, 2H); ${ }^{13} \mathrm{C}$ NMR (75 MHz, DMSO-d $\left.d_{6}\right) \delta: 160.9$, $159.8,154.5,150.5,137.3,131.9,130.8,128.4,127.5$, $127.0,124.7,121.8,121.7,117.7,115.3,113.8,113.4$, 110.6, 99.6, 55.2, 31.0, 26.1; IR (KBr) v: 2948, 2841, 1652, 1510, 1242, 1028, 1002, 821, $755 \mathrm{~cm}^{-1}$; HRMS (MALDI) calcd for $\mathrm{C}_{25} \mathrm{H}_{19} \mathrm{ClNO}_{4}[\mathrm{M}+\mathrm{H}]^{+}$432.0997, found 432.0999 .

10,12-二氯-8-(4-氟苯基)-2,14-二氢- $1 H-8,14$-亚甲基 苯并 $[7,8][1,3]$ 二氧桥 $[5,4-c]$ 喹啉-1-酮(3o): 白色固体 148 $\mathrm{mg}$, 产率 65\%. m.p. $>300{ }^{\circ} \mathrm{C}$; ${ }^{1} \mathrm{H} \mathrm{NMR}(300 \mathrm{MHz}$, DMSO-d $\left.d_{6}\right) \delta: 11.74(\mathrm{~s}, 1 \mathrm{H}), 7.90(\mathrm{t}, J=7.3 \mathrm{~Hz}, 2 \mathrm{H}), 7.81$ $(\mathrm{d}, J=7.9 \mathrm{~Hz}, 1 \mathrm{H}), 7.57 \sim 7.51(\mathrm{~m}, 2 \mathrm{H}), 7.43 \sim 7.32(\mathrm{~m}$, $4 \mathrm{H}), 7.24 \sim 7.19(\mathrm{~m}, 1 \mathrm{H}), 4.43(\mathrm{~s}, 1 \mathrm{H}), 2.65 \sim 2.46(\mathrm{~m}$, $2 \mathrm{H}) ;{ }^{13} \mathrm{C}$ NMR $\left(75 \mathrm{MHz}, \mathrm{DMSO}-d_{6}\right) \delta: 162.6\left(\mathrm{~d},{ }^{1} J_{\mathrm{C}-\mathrm{F}}=\right.$ $244.5 \mathrm{~Hz}), 160.8,154.2,146.4,137.4,135.5\left(\mathrm{~d},{ }^{4} J_{\mathrm{C}-\mathrm{F}}=2.9\right.$ $\mathrm{Hz}), 131.0,129.7,128.2\left(\mathrm{~d},{ }^{3} J_{\mathrm{C}-\mathrm{F}}=8.6 \mathrm{~Hz}\right), 127.5,126.1$, $125.0,121.9,121.8,120.9,115.46\left(\mathrm{~d},{ }^{2} J_{\mathrm{C}-\mathrm{F}}=21.6 \mathrm{~Hz}\right)$, $115.38,113.2,110.0,99.9,30.5$, 26.2; IR (KBr) v: 3498, 2947, 2411, 1652, 1507, 1292, 1099, 834, $752 \mathrm{~cm}^{-1}$; HRMS (MALDI) calcd for $\mathrm{C}_{24} \mathrm{H}_{15} \mathrm{Cl}_{2} \mathrm{FNO}_{3}[\mathrm{M}+\mathrm{H}]^{+}$ 454.0408, found 454.0398.

2-甲基-8-苯基-2,14-二氢- $1 H-8,14$ - 亚甲基苯并 [7,8][1,3]二氧桥[5,4-c]喹啉-1-酮(3p): 白色固体 $143 \mathrm{mg}$, 产率 73\%. m.p. $185 \sim 186{ }^{\circ} \mathrm{C}$; ${ }^{1} \mathrm{H}$ NMR $(300 \mathrm{MHz}$, $\left.\mathrm{CDCl}_{3}\right) \delta: 8.09 \sim 8.06(\mathrm{~m}, 1 \mathrm{H}), 7.82 \sim 7.78(\mathrm{~m}, 2 \mathrm{H})$, $7.63 \sim 7.60(\mathrm{~m}, 1 \mathrm{H}), 7.58 \sim 7.46(\mathrm{~m}, 4 \mathrm{H}), 7.32(\mathrm{~d}, J=8.5$ $\mathrm{Hz}, 1 \mathrm{H}), 7.23 \sim 7.13(\mathrm{~m}, 2 \mathrm{H}), 7.05 \sim 6.93(\mathrm{~m}, 2 \mathrm{H}), 4.60 \sim$ $4.58(\mathrm{~m}, 1 \mathrm{H}), 3.71(\mathrm{~s}, 3 \mathrm{H}), 2.48 \sim 2.33(\mathrm{~m}, 2 \mathrm{H}) ;{ }^{13} \mathrm{C} \mathrm{NMR}$ 
$\left(75 \mathrm{MHz}, \mathrm{CDCl}_{3}\right) \delta: 161.7,154.4,151.8,140.6,138.6$, $130.8,129.1,128.5,128.2,127.9,126.1,125.8,123.1$, $121.9,121.7,116.1,115.5,114.0,111.1,99.5,33.2,29.4$, 27.4; IR (KBr) v: 3434, 1638, 1490, 1397, 1271, 1116, 1038, 959, $751 \mathrm{~cm}^{-1}$; HRMS (MALDI) calcd for $\mathrm{C}_{25} \mathrm{H}_{20} \mathrm{NO}_{3}[\mathrm{M}+\mathrm{H}]^{+}$382.1438, found 382.1438.

2-甲基-8-(4-甲基苯基)-2,14-二氢- $1 H$-8,14-亚甲基 苯并 $[7,8][1,3]$ 二氧桥 [5,4-c]喹啉-1-酩 (3q): 白色固体 $148 \mathrm{mg}$, 产率 75\%. m.p. $191 \sim 192{ }^{\circ} \mathrm{C} ;{ }^{1} \mathrm{H}$ NMR $(300$ $\left.\mathrm{MHz}, \mathrm{CDCl}_{3}\right) \delta: 8.06(\mathrm{~d}, J=8.1 \mathrm{~Hz}, 1 \mathrm{H}), 7.68$ (d, $J=7.8$ $\mathrm{Hz}, 2 \mathrm{H}), 7.61(\mathrm{~d}, J=7.0 \mathrm{~Hz}, 1 \mathrm{H}), 7.54(\mathrm{t}, J=8.6 \mathrm{~Hz}, 1 \mathrm{H})$, $7.31(\mathrm{~d}, J=7.8 \mathrm{~Hz}, 3 \mathrm{H}), 7.22 \sim 7.13(\mathrm{~m}, 2 \mathrm{H}), 7.04 \sim 6.93$ (m, 2H), $4.58(\mathrm{~s}, 1 \mathrm{H}), 3.70(\mathrm{~s}, 3 \mathrm{H}), 2.43 \sim 2.32(\mathrm{~m}, 5 \mathrm{H})$; ${ }^{13} \mathrm{C}$ NMR $\left(75 \mathrm{MHz}, \mathrm{CDCl}_{3}\right) \delta: 161.7,154.5,151.9,139.0$, 138.6, 137.7, 130.8, 129.2, 128.2, 127.9, 126.1, 125.7, 123.1, 121.8, 121.6, 116.1, 115.5, 113.9, 111.0, 99.6, 33.2, 29.4, 27.4, 21.2; IR (KBr) v: 3434, 1636, 1466, 1395, 1270, 1180, 1004, 956, $755 \mathrm{~cm}^{-1}$; HRMS (MALDI) calcd for $\mathrm{C}_{26} \mathrm{H}_{22} \mathrm{NO}_{3}[\mathrm{M}+\mathrm{H}]^{+}$396.1594, found 396.1595.

6-苯基-2,12-二氢- $1 H$-6, 12-亚甲基苯并 [7,8][1,3]二 氧桥[5,4-c]吡啶-1-酮(3r): 白色固体 $124 \mathrm{mg}$, 产率 78\%. m.p. $246 \sim 248{ }^{\circ} \mathrm{C} ;{ }^{1} \mathrm{H}$ NMR (300 MHz, $\mathrm{CDCl}_{3}$ ) $\delta: 11.28$ (s, 1H), $7.73 \sim 7.69(\mathrm{~m}, 2 \mathrm{H}), 7.55 \sim 7.52(\mathrm{~m}, 1 \mathrm{H}), 7.50 \sim$ $7.42(\mathrm{~m}, 3 \mathrm{H}), 7.18 \sim 7.12(\mathrm{~m}, 2 \mathrm{H}), 7.03(\mathrm{~d}, J=8.1 \mathrm{~Hz}$, $1 \mathrm{H}), 6.96 \sim 6.91(\mathrm{~m}, 1 \mathrm{H}), 6.12 \sim 6.09(\mathrm{~m}, 1 \mathrm{H}), 4.43 \sim 4.42$ (m, $1 \mathrm{H}), 2.40 \sim 2.26(\mathrm{~m}, 2 \mathrm{H}) ;{ }^{13} \mathrm{C} \mathrm{NMR}\left(75 \mathrm{MHz}, \mathrm{CDCl}_{3}\right)$ $\delta: 163.7,161.2,151.7,140.3,132.5,129.1,128.4,128.0$, 127.9, 126.1, 125.6, 121.6, 116.2, 112.2, 100.8, 99.4, 32.7, 26.4; IR (KBr) v: 3436, 2361, 1642, 1558, 1465, 1119, $1004,1029,755 \mathrm{~cm}^{-1}$; HRMS (MALDI) calcd for $\mathrm{C}_{20} \mathrm{H}_{16^{-}}$ $\mathrm{NO}_{3}[\mathrm{M}+\mathrm{H}]^{+} 318.1125$, found 318.1123 .

3- 甲基-6-苯基-2,12-二氢-1H-6,12-亚甲基苯并 [7,8][1,3]二氧桥[5,4-c]吡啶-1-酮(3s): 白色固体 $113 \mathrm{mg}$, 产率 68\%. m.p. $277 \sim 279{ }^{\circ} \mathrm{C} ;{ }^{1} \mathrm{H}$ NMR $(300 \mathrm{MHz}$, $\left.\mathrm{CDCl}_{3}\right) \delta: 12.19(\mathrm{~s}, 1 \mathrm{H}), 7.72 \sim 7.69(\mathrm{~m}, 2 \mathrm{H}), 7.51 \sim 7.42$ $(\mathrm{m}, 4 \mathrm{H}), 7.16 \sim 7.10(\mathrm{~m}, 1 \mathrm{H}), 7.01(\mathrm{~d}, J=7.5 \mathrm{~Hz}, 1 \mathrm{H})$, $6.92 \sim 6.86(\mathrm{~m}, 1 \mathrm{H}), 5.90(\mathrm{~s}, 1 \mathrm{H}), 4.39 \sim 4.37(\mathrm{~m}, 1 \mathrm{H})$, $2.32 \sim 2.28(\mathrm{~m}, 5 \mathrm{H}) ;{ }^{13} \mathrm{C} \mathrm{NMR}\left(75 \mathrm{MHz}, \mathrm{CDCl}_{3}\right) \delta: 164.2$, $161.4,151.8,143.6,140.5,129.0,128.4,127.8,127.7$, $126.5,125.6,121.4,116.2,108.7,99.4,99.3,33.0,26.3$, 19.0; IR (KBr) v: 3423, 2361, 1632, 1449, 1255, 1178, 1109, 1026, 797, $757 \mathrm{~cm}^{-1}$; HRMS (MALDI) calcd for $\mathrm{C}_{21} \mathrm{H}_{18} \mathrm{NO}_{3}[\mathrm{M}+\mathrm{H}]^{+}$332.1281, found 332.1280.

4-乙酯基-6-苯基-2,12-二氢-1 $H$-6, 12 -亚甲基苯并 [7,8][1,3]二氧桥[5,4-c]吡啶-1-酮(3t): 白色固体 $148 \mathrm{mg}$,
产率 76\%. m.p. 244 $246{ }^{\circ} \mathrm{C} ;{ }^{1} \mathrm{H}$ NMR $(300 \mathrm{MHz}$, $\left.\mathrm{CDCl}_{3}\right) \delta: 12.09(\mathrm{~s}, 1 \mathrm{H}), 8.11(\mathrm{~s}, 1 \mathrm{H}), 7.87 \sim 7.84(\mathrm{~m}, 2 \mathrm{H})$, $7.55 \sim 7.44(\mathrm{~m}, 4 \mathrm{H}), 7.21 \sim 7.15(\mathrm{~m}, 1 \mathrm{H}), 7.03 \sim 6.93(\mathrm{~m}$, $2 \mathrm{H}), 4.46 \sim 4.44(\mathrm{~m}, 1 \mathrm{H}), 4.41 \sim 4.26(\mathrm{~m}, 2 \mathrm{H}), 2.51 \sim 2.22$ $(\mathrm{m}, 2 \mathrm{H}), 1.35(\mathrm{t}, J=7.1 \mathrm{~Hz}, 3 \mathrm{H}) ;{ }^{13} \mathrm{C}$ NMR $(75 \mathrm{MHz}$, $\left.\mathrm{CDCl}_{3}\right) \delta: 163.5,163.3,159.5,151.7,140.1,139.3,129.1$, $128.4,128.1,128.0,126.0,125.5,121.7,116.3,112.1$, 105.3, 99.9, 61.1, 32.2, 26.4, 14.3; IR (KBr) v: 2857, 2363, 1726, 1637, 1440, $1285 \mathrm{~cm}^{-1}$; HRMS (MALDI) calcd for $\mathrm{C}_{23} \mathrm{H}_{20} \mathrm{NO}_{5}[\mathrm{M}+\mathrm{H}]^{+}$390.1336, found 390.1341.

4- 氯-6- 苯基-2,12-二氢-1H-6,12- 亚甲基苯并 [7,8][1,3]二氧桥[5,4-c]吡啶-1-酮(3u): 白色固体 $114 \mathrm{mg}$, 产率 65\%. m.p. $>300{ }^{\circ} \mathrm{C} ;{ }^{1} \mathrm{H}$ NMR $\left(300 \mathrm{MHz}, \mathrm{DMSO}-d_{6}\right)$ $\delta: 11.69(\mathrm{~s}, 1 \mathrm{H}), 7.75 \sim 7.72(\mathrm{~m}, 2 \mathrm{H}), 7.55 \sim 7.47(\mathrm{~m}, 4 \mathrm{H})$, 7.39 (dd, $J=7.5,1.5 \mathrm{~Hz}, 1 \mathrm{H}), 7.21 \sim 7.15(\mathrm{~m}, 1 \mathrm{H}), 7.02 \sim$ $6.92(\mathrm{~m}, 2 \mathrm{H}), 4.28 \sim 4.26(\mathrm{~m}, 1 \mathrm{H}), 2.47 \sim 2.34(\mathrm{~m}, 2 \mathrm{H})$; ${ }^{13} \mathrm{C}$ NMR (75 MHz, DMSO- $\left.d_{6}\right) \delta: 160.3,155.1,151.1$, 139.7, 132.1, 129.1, 128.4, 127.9, 127.7, 126.0, 125.5, 121.4, 115.9, 112.5, 103.3, 99.6, 31.1, 26.1; IR (KBr) v: 3434, 2760, 1651, 1483, 1246, 1163, 1076, 928, $757 \mathrm{~cm}^{-1}$; HRMS (MALDI) calcd for $\mathrm{C}_{20} \mathrm{H}_{15} \mathrm{ClNO}_{3}[\mathrm{M}+\mathrm{H}]^{+}$ 352.0735 , found 352.0731 .

\subsection{2目标化合物 $\mathbf{5 / 6}$ 的合成}

将 8-苯基-2,14-二氢- $1 H$ - 8 , 14-亚甲基苯并 $[7,8][1,3]$ 二氧桥[5,4-c]喹啉-1-酮 (3a) $(0.5 \mathrm{mmol})$ 和溴代物 4 (1.5 $\mathrm{mmol}$ )加入到 $25 \mathrm{~mL}$ 耐压管中, 以 3.0 equiv. 的碳酸钾为 碱, 再向其中加入 $N, N$-二甲基甲酰胺(DMF) $(5 \mathrm{~mL}$ ), 当 反应物消失后( $3 \sim 8 \mathrm{~h}$, 用 TLC 监测), 将混合物冷却至 室温, 减压除去溶剂, 加入 $30 \mathrm{~mL}$ 水后用乙酸乙酯萃取 $(20 \mathrm{~mL} \times 3)$, 合并有机相后干燥、过滤, 再减压除去乙酸 乙酯后, 剩余物以石油醚和乙酸乙酯为洗脱剂(体积比 为 $5: 1 \sim 20: 1)$, 通过硅胶柱层析分离得到目标化合 物.

2-乙基-8- 苯基-2,14-二氢- $1 H-8,14$ - 亚甲基苯并 [7,8][1,3]二氧桥[5,4-c]喹啉-1-酮(5a): 白色固体 $119 \mathrm{mg}$, 产率 $60 \%$. m.p. $210 \sim 212{ }^{\circ} \mathrm{C} ;{ }^{1} \mathrm{H}$ NMR $(300 \mathrm{MHz}$, $\left.\mathrm{CDCl}_{3}\right) \delta: 8.07(\mathrm{dd}, J=8.0,1.2 \mathrm{~Hz}, 1 \mathrm{H}), 7.81 \sim 7.78(\mathrm{~m}$, $2 \mathrm{H}), 7.64 \sim 7.61(\mathrm{~m}, 1 \mathrm{H}), 7.54 \sim 7.46(\mathrm{~m}, 4 \mathrm{H}), 7.31(\mathrm{~d}, J=$ $8.5 \mathrm{~Hz}, 1 \mathrm{H}), 7.22 \sim 7.14(\mathrm{~m}, 2 \mathrm{H}), 7.04(\mathrm{~d}, J=7.7 \mathrm{~Hz}, 1 \mathrm{H})$, $6.99 \sim 6.94(\mathrm{~m}, 1 \mathrm{H}), 4.60 \sim 4.58(\mathrm{~m}, 1 \mathrm{H}), 4.44 \sim 4.23(\mathrm{~m}$, $2 \mathrm{H}), 2.46 \sim 2.32(\mathrm{~m}, 2 \mathrm{H}), 1.33(\mathrm{t}, J=7.1 \mathrm{~Hz}, 3 \mathrm{H}) ;{ }^{13} \mathrm{C}$ NMR $\left(75 \mathrm{MHz}, \mathrm{CDCl}_{3}\right) \delta: 161.1,154.3,151.8,140.6$, $137.5,130.7,129.1,128.5,128.3,127.8,126.1,125.7$, 123.2, 121.64, 121.61, 116.1, 115.6, 113.8, 110.9, 99.5, 37.3, 33.2, 27.3, 12.9; IR (KBr) v: 3441, 1632, 1451, 1393, 
1232, 1130, 892, 760, $696 \mathrm{~cm}^{-1}$; HRMS (MALDI) calcd for $\mathrm{C}_{26} \mathrm{H}_{22} \mathrm{NO}_{3}[\mathrm{M}+\mathrm{H}]^{+}$396.1594, found 396.1597.

2- 苄基-8-苯基-2,14-二氢- $1 H-8,14$ - 亚甲基苯并 [7,8][1,3]二氧桥[5,4-c]喹啉-1-酮(5b): 白色固体 $119 \mathrm{mg}$, 产率 52\%. m.p. 223 224 ${ }^{\circ} \mathrm{C}$; ${ }^{1} \mathrm{H}$ NMR $(300 \mathrm{MHz}$, $\left.\mathrm{CDCl}_{3}\right) \delta: 8.07(\mathrm{dd}, J=1.3,7.9 \mathrm{~Hz}, 1 \mathrm{H}), 7.81(\mathrm{dd}, J=1.8$, $8.1 \mathrm{~Hz}, 2 \mathrm{H}), 7.63$ (dd, $J=1.5,7.5 \mathrm{~Hz}, 1 \mathrm{H}), 7.54 \sim 7.46(\mathrm{~m}$, $3 \mathrm{H}), 7.41 \sim 7.36(\mathrm{~m}, 2 \mathrm{H}), 7.31 \sim 7.26(\mathrm{~m}, 2 \mathrm{H}), 7.22 \sim 7.14$ $(\mathrm{m}, 5 \mathrm{H}), 7.06(\mathrm{~d}, J=7.8 \mathrm{~Hz}, 1 \mathrm{H}), 7.00 \sim 6.97(\mathrm{~m}, 1 \mathrm{H})$, $5.74(\mathrm{~d}, J=16.2 \mathrm{~Hz}, 1 \mathrm{H}), 5.32(\mathrm{~d}, J=15.9 \mathrm{~Hz}, 1 \mathrm{H}), 4.66 \sim$ $4.64(\mathrm{~m}, 1 \mathrm{H}), 2.50 \sim 2.37(\mathrm{~m}, 2 \mathrm{H}) ;{ }^{13} \mathrm{C}$ NMR $(75 \mathrm{MHz}$, $\left.\mathrm{CDCl}_{3}\right) \delta: 161.9,154.8,151.8,140.6,138.1,136.7,130.8$, $129.1,128.7,128.5,128.3,127.9,127.1,126.5,126.1$, $125.8,123.1,122.0,121.8,116.2,115.7,114.8,110.9$, 99.7, 46.1, 33.2, 27.4; IR (KBr) v: 3431, 2923, 1632, 1597, 1454, 1131, 1006, 753, $697 \mathrm{~cm}^{-1}$; HRMS (MALDI) calcd for $\mathrm{C}_{31} \mathrm{H}_{24} \mathrm{NO}_{3}[\mathrm{M}+\mathrm{H}]^{+}$458.1751, found 458.1747.

1-乙氧基- 8 -苯基- $14 H-8,14$-亚甲基苯并 $[7,8][1,3]$ 二 氧桥[5,4-c]喹啉(6a): 白色固体 $24 \mathrm{mg}$, 产率 12\%. m.p. 216 $217{ }^{\circ} \mathrm{C} ;{ }^{1} \mathrm{H}$ NMR $\left(300 \mathrm{MHz}, \mathrm{CDCl}_{3}\right) \delta: 8.13(\mathrm{~d}, J=$ $8.3 \mathrm{~Hz}, 1 \mathrm{H}), 7.84 \sim 7.81(\mathrm{~m}, 2 \mathrm{H}), 7.73(\mathrm{~d}, J=8.1 \mathrm{~Hz}, 1 \mathrm{H})$, $7.58 \sim 7.44(\mathrm{~m}, 5 \mathrm{H}), 7.33(\mathrm{t}, J=7.0 \mathrm{~Hz}, 1 \mathrm{H}), 7.18 \sim 7.12$ $(\mathrm{m}, 1 \mathrm{H}), 7.04(\mathrm{~d}, J=7.5 \mathrm{~Hz}, 1 \mathrm{H}), 6.96 \sim 6.92(\mathrm{~m}, 1 \mathrm{H})$, $4.66 \sim 4.56(\mathrm{~m}, 2 \mathrm{H}), 4.55 \sim 4.53(\mathrm{~m}, 1 \mathrm{H}), 2.49 \sim 2.38(\mathrm{~m}$, 2H), $1.57 \sim 1.53(\mathrm{~m}, 3 \mathrm{H}) ;{ }^{13} \mathrm{C}$ NMR $\left(75 \mathrm{MHz}, \mathrm{CDCl}_{3}\right) \delta$ : $159.6,155.1,152.1,145.6,140.9,129.4,129.0,128.5$, $127.9,127.8,126.8,126.2,125.8,123.4,121.6,121.4$, $118.2,116.4,106.8,99.3,62.0,33.1,27.0,14.8$; IR (KBr) $v: 3431,2922,1631,1401,1347,1116,887,755,696$ $\mathrm{cm}^{-1}$; HRMS (MALDI) calcd for $\mathrm{C}_{26} \mathrm{H}_{22} \mathrm{NO}_{3}[\mathrm{M}+\mathrm{H}]^{+}$ 396.1594, found 396.1598.

1-芐氧基-8-苯基- $14 H$-8, 14-亚甲基苯并 [7,8][1,3]二 氧桥[5,4-c]喹啉 $(6 \mathbf{b})$ : 白色固体 $30 \mathrm{mg}$, 产率 13\%. m.p. $234 \sim 236{ }^{\circ} \mathrm{C} ;{ }^{1} \mathrm{H}$ NMR $\left(300 \mathrm{MHz}, \mathrm{CDCl}_{3}\right) \delta: 8.15(\mathrm{~d}, J=$ $8.0 \mathrm{~Hz}, 1 \mathrm{H}), 7.83 \sim 7.76(\mathrm{~m}, 3 \mathrm{H}), 7.60(\mathrm{~d}, J=7.5 \mathrm{~Hz}, 3 \mathrm{H})$, $7.48(\mathrm{t}, J=7.6 \mathrm{~Hz}, 4 \mathrm{H}), 7.33 \sim 7.30(\mathrm{~m}, 4 \mathrm{H}), 7.13(\mathrm{t}, J=$ $7.7 \mathrm{~Hz}, 1 \mathrm{H}), 7.03$ (d, $J=7.8 \mathrm{~Hz}, 1 \mathrm{H}), 6.86(\mathrm{t}, J=7.2 \mathrm{~Hz}$, 1H), 5.56 (s, 2H), $4.54(\mathrm{~s}, 1 \mathrm{H}), 2.41(\mathrm{~s}, 2 \mathrm{H}) ;{ }^{13} \mathrm{C}$ NMR $(75$ $\left.\mathrm{MHz}, \mathrm{CDCl}_{3}\right) \delta: 159.3,155.5,152.1,145.5,140.9,137.5$, $129.6,129.0,128.5,128.4,127.9,126.9,126.0,125.8$, $123.6,121.7,121.4,118.4,116.4,106.8,99.4,68.1,33.1$, 27.0; IR (KBr) v: 3441, 1629, 1417, 1340, 1103, 999, 872, $760,690 \mathrm{~cm}^{-1}$; HRMS (MALDI) calcd for $\mathrm{C}_{31} \mathrm{H}_{24} \mathrm{NO}_{3}$ $[\mathrm{M}+\mathrm{H}]^{+}$458.1751, found 458.1749.

1-异丙氧基-8-苯基-14H-8,14-亚甲基苯并 $[7,8][1,3]$ 二氧桥[5,4-c]喹啉(6c): 白色固体 $137 \mathrm{mg}$, 产率 67\%. m.p. $218 \sim 220{ }^{\circ} \mathrm{C} ;{ }^{1} \mathrm{H}$ NMR $\left(300 \mathrm{MHz}, \mathrm{CDCl}_{3}\right) \delta: 8.05 \sim$ $8.02(\mathrm{~m}, 1 \mathrm{H}), 7.75 \sim 7.72(\mathrm{~m}, 2 \mathrm{H}), 7.63(\mathrm{~d}, J=8.0 \mathrm{~Hz}$, $1 \mathrm{H}), 7.48 \sim 7.42(\mathrm{~m}, 3 \mathrm{H}), 7.40 \sim 7.37(\mathrm{~m}, 1 \mathrm{H}), 7.25 \sim 7.20$ $(\mathrm{m}, 1 \mathrm{H}), 7.15 \sim 7.03(\mathrm{~m}, 2 \mathrm{H}), 6.96 \sim 6.94(\mathrm{~m}, 1 \mathrm{H}), 6.88 \sim$ $6.82(\mathrm{~m}, 1 \mathrm{H}), 5.59 \sim 5.51(\mathrm{~m}, 1 \mathrm{H}), 4.44 \sim 4.42(\mathrm{~m}, 1 \mathrm{H})$, $2.34 \sim 2.32(\mathrm{~m}, 2 \mathrm{H}) 1.44(\mathrm{~d}, J=6.2 \mathrm{~Hz}, 3 \mathrm{H}), 1.37(\mathrm{~d}, J=$ $6.2 \mathrm{~Hz}, 3 \mathrm{H}) ;{ }^{13} \mathrm{C} \mathrm{NMR}\left(75 \mathrm{MHz}, \mathrm{CDCl}_{3}\right) \delta: 159.0,155.1$, $152.2,145.6,140.9,129.3,129.0,128.5,127.9,127.8$, $126.8,126.2,125.8,123.2,121.5,121.3,118.0,116.4$, 107.0, 99.2, 68.3, 33.0, 26.9, 22.4, 22.3; IR (KBr) v: 3439, 2968, 1948, 1624, 1417, 1100, 876, 804, $753 \mathrm{~cm}^{-1}$; HRMS (MALDI) calcd for $\mathrm{C}_{27} \mathrm{H}_{24} \mathrm{NO}_{3}[\mathrm{M}+\mathrm{H}]^{+} 410.1751$, found 410.1753 .

辅助材料(Supporting Information) 化合物 $\mathbf{3 a} \sim \mathbf{3 u}$, $5 a, 5 b$ 和 $6 a \sim 6 c$ 的 ${ }^{1} \mathrm{H} N \mathrm{NMR}$ 和 ${ }^{13} \mathrm{C} \mathrm{NMR}$ 图谱, 3r 和 $6 c$ 的晶体结构数据. 这些材料可以免费从本刊网站 (http://sioc-journal.cn/)上下载.

\section{References}

[1] Neve, J. E.; Wijesekera, H. P.; Duffy, S.; Jenkins, I. D.; Ripper, J. A.; Teague, S. J.; Campitelli, M.; Garavelas, A.; Nikolakopoulos, G.; Le, P. V.; de A. Leone, P.; Pham, N. B.; Shelton, P.; Fraser, N.; Carroll, A. R.; Avery, V. M.; McCrae, C.; Williams, N.; Quinn, R. J. J. Med. Chem. 2014, 57, 1252.

[2] Zhao, S.; Huang, J.; Cheng, J.; Liu, B.; Chen, C. Chin. J. Org. Chem. 2012, 32, 651 (in Chinese). (赵圣印, 黄婧, 程健, 刘保硕, 陈晨, 有机化学, 2012, 32, 651.)

[3] Banbury, L. K.; Shou, Q.; Renshaw, D. E.; Lambley, E. H.; Griesser, H. J.; Mon H.; Wohlmuth, H. J. Ethnopharmacol. 2015, 163, 251.

[4] Ahsan, M.; Haque, M. R.; Hossain, M. B.; Islam, S. N.; Gray, A. I.; Hasan, C. M. Phytochemistry 2014, 103, 8.

[5] Tedesco, R.; Shaw, A. N.; Bambal, R.; Chai, D.; Concha, N. O.; Darcy, M. G.; Dhanak, D.; Fitch, D. M.; Gates, A.; Gerhardt, W. G.; Halegoua, D. L.; Han, C.; Hofmann, G. A.; Johnston, V. K.; Kaura, A. C.; Liu, N.; Keenan, R. M.; Lin-Goerke, J.; Sarisky, R. T.; Wiggall, K. J.; Zimmerman, M. N.; Duffy, K. J. J. Med. Chem. 2006, 49, 971.

[6] Chen, T.; Luo, Y.; Hu, Y.; Yang, B.; Lu, W. Eur. J. Med. Chem. 2013, 64, 613.

[7] Detsi, A.; Bouloumbasi, D.; Prousis, K. C.; Koufaki, M.; Athanasellis, G.; Melagraki, G.; Afantitis, A.; Lgglessi-Markopoulou, O.; Kontogiorgis, C.; Hadjipavlou-Litina, D. J. J. Med. Chem. 2007, $50,2450$.

[8] Nishino, H.; Kumabe, R.; Hamada, R.; Yakut, M. Tetrahedron 2014, 70, 1437.

[9] Lei, M.; Ma, L.; Hu, L. Tetrahedron Lett. 2011, 52, 2597.

[10] Pei, W.; Deng, Q.; Wang, H.; Sun L. Chin. J. Org. Chem. 2006, 26, 364 (in Chinese)

(裴文, 邓琼, 王海滨, 孙莉, 有机化学, 2006, 26, 364.)

[11] Presset, M.; Coquerel, Y.; Rodriguez, J. Chem. Rev. 2013, 113, 525.

[12] Ruiz, M.; López-Alvarado, P.; Giorgi, G.; Menéndez, J. C. Chem. 
Soc. Rev. 2011, 40, 3445.

[13] Chen, D.; Kuo, P.; Yang, D. Bioorg. Med. Chem. Lett. 2005, 15, 2665.

[14] He, Z.; Yang, X.; Jiang, W. Org. Lett. 2015, 17, 3880.

[15] Wang, F.; Chen, F.; Qu, M.; Li, T.; Liu, Y.; Shi, M. Chem. Commun. 2013, 49,3360 .

[16] Reddy, G. M.; Sridhar, P. R. Eur. J. Org. Chem. 2014, 1496.

[17] Xia, L.; Cai, H.; Lee, Y. R. Org. Biomol. Chem. 2014, 12, 4386.

[18] Jiang, X.; Song, Z.; Xu, C.; Yao, Q.; Zhang, A. Eur. J. Org. Chem. 2014, 418.

[19] Srinivas, V.; Koketsu, M. J. Org. Chem. 2013, 78, 11612.

[20] Ganguly, N. C.; Roy, S.; Mondal, P. RSC Adv. 2014, 4, 42078.

[21] Yin, G.; Ren, T.; Rao, Y.; Zhou, Y.; Li, Z.; Shu, W.; Wu, A. J. Org. Chem. 2013, 78, 3132.

[22] Rao, Y.; Yin, G. Org. Biomol. Chem. 2013, 11, 6029.

[23] Rao, Y.; Liu, M.; Wu, L.; Yin, G. RSC Adv. 2014, 4, 64551.
[24] Bingi, C.; Emmadi, N. R.; Chennapuram, M.; Nanubolu, J. B.; Atmakur, K. RSC Adv. 2014, 4, 35009.

[25] Yin, G.; Fan, L.; Ren, T.; Zheng, C.; Tao, Q.; Wu, A.; She, N. Org. Biomol. Chem. 2012, 10, 8877.

[26] Kinoshita, T.; Nashimoto, A.; Yamamura, Y.; Okamura, T.; Sasako, M.; Sakamoto, J.; Kojima, H.; Hiratsuka, M.; Arai, K.; Sairenji, M.; Fukushima, N.; Kimura, H.; Nakajima, T. Gastric Cancer 2004, 7, 104.

[27] Luiza, B. D. O.; Borgati, T. F.; de Freitas, R. P.; Ruiz, A. L.; Marchetti, G. M.; de Carvalho, J. E.; da Cunha, E. F. F.; Ramalho, T. C.; Alves, R. B. Eur. J. Med. Chem. 2014, 84, 595.

[28] Hao, X.; Xu, Z.; Lu, H.; Dai, X.; Yang, T.; Lin, X.; Ren, F. Org. Lett. 2015, 17, 3382.

[29] Torhan, M. C.; Peet, N. P.; Williams, J. D. Tetrahedron Lett. 2013, $54,3926$. 